\title{
Atrioventricular Block of Dyasautonomic Origin Complicated by Ventricular Asystole: A Case Report
}

Safae Harrak ${ }^{1 *}$, Souad Aboudrar ${ }^{2}$, Mustapha El bakkali ${ }^{2}$, Halima Benjelloun ${ }^{1}$

${ }^{1}$ Cadiology A Department, Ibn Sina Hospital, Mohammed V University in Rabat, Morocco

${ }^{2}$ Physiology of Exercise Team and Autonomic Nervous System (PET-ANS), Faculty of Medicine and Pharmacy, Mohammed V University in Rabat, Morocco

DOI: $10.36347 /$ sjams.2020.v08i08.0027

| Received: 07.08.2020 | Accepted: 14.08.2020 | Published: 30.08.2020

*Corresponding author: Safae Harrak

Abstract

Atrioventricular (AV) block is an interruption or delay to the electrical conduction due to conduction system abnormalities in the atrioventricular node or in the His-Purkinje system. In addition to intrinsic innervation, the conduction system receives an extrinsic innervation from the two contingents of the autonomic nervous system. In this paper, we present this case on atrioventricular block complicated by asystole owing to increased vagal tone to provide an insight into the contribution of the autonomic nervous system in the etiopathogenesis of conduction disorders.

Keywords: Atrioventricular (AV), asystole, nervous system, etiopathogenesis.

Copyright @ 2020: This is an open-access article distributed under the terms of the Creative Commons Attribution license which permits unrestricted use, distribution, and reproduction in any medium for non-commercial use (NonCommercial, or CC-BY-NC) provided the original author and source are credited.

\section{INTRODUCTION}

Atrioventricular (AV) block is interruption or delay of electrical conduction due to conduction system abnormalities in the atrioventricular node or the HisPurkinje system. We present a case report on atrioventricular block complicated by asystole owing to increased vagal tone to provide an insight into the contribution of the autonomic nervous system in the etiopathogenesis of conduction disorders.

\section{Case Report}

A 51-year-old women without medical history, was admitted for weakness, orthostatic intolerance and palpitations especially at night that started 6 months ago. Physical examination, electrocardiogram and transthoracic echocardiogram did not show any abnormalities. 24-hour Holter monitoring objectified paroxysmal asymptomatic second-degree atrioventricular block. She was referred to cardiology A department unit to explore her autonomic nervous system. She was initially placed in calm. We measured blood pressure, heart rate on admission and we did baseline electrocardiogram. Then, we preceded to cardiovascular autonomic tests interspersed with periods of rest, as describing by Ewing David [1, 2] and detailed by Phillip Low [3].

In the Deep Breathing test, we ask the patient to breathe deeply at a frequency of six breaths for one minute. It evaluates the vagal response. Electrocardiogram have showed, few second after the beginning, a first degree atrioventricular block (PR from $0,2 \mathrm{~s}$ to $0.32 \mathrm{~s}$ ) (See Figure-1), then a passage in complete atrio-ventricular block (See Figure-2), then, a ventricular asystole during 3.2se (See Figure-3) reasons for stopping the test. Clinically, the patient hadn't felt any symptoms.

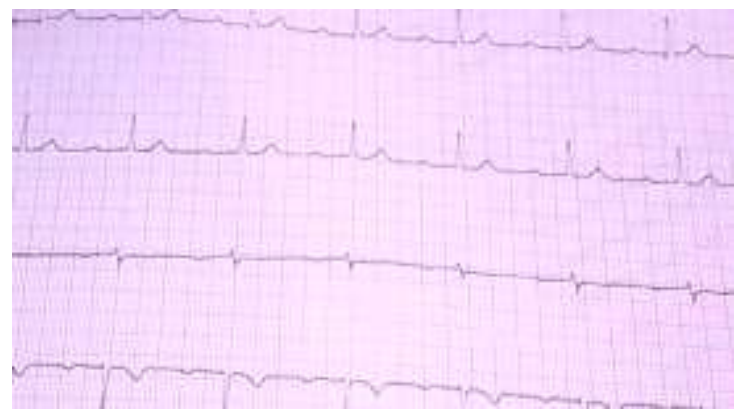

Figure-1: Electrocardiogram showing passage to first degree atrioventricular block (PR space at 0.32seconds) 


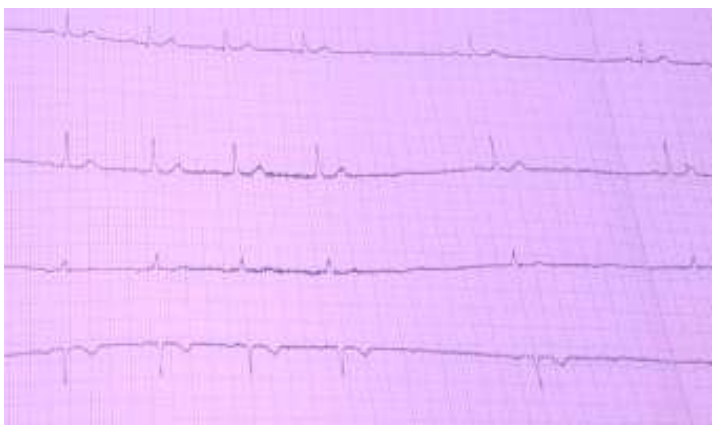

Figure-2: Electrocardiogram showing progression to complete atrioventricular block (30beat/min)

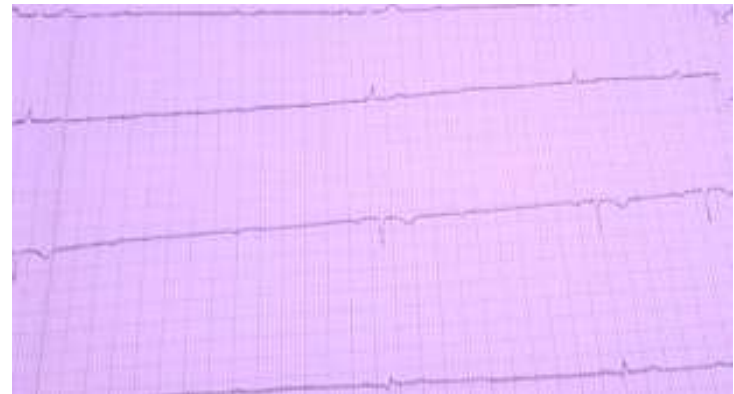

Figure-3: Electrocardiogram showing progression to ventricular asystole of 3,2 seconds

We then carried out the hand grip test, which consists of the patient applying a maximum manual pressure of $50 \%$ assisted by a dynamometer. It calculates peripheral sympathetic response $\alpha$ and can also evaluate vagal response. The mental stress test is based on doing arithmetic calculations and evaluate the central sympathetic nerves activities. We have obtained a value of $9 \%$ in favor of $\alpha$ deficiency and $7 \%$ in favor $\beta$ central sympathetic deficiency. The Table- 1 summarizes the results from the different tests and interpretation of each one.

Table-1: Results of different tests and interpretation of each one.

\begin{tabular}{llcl}
\hline Test & \multicolumn{1}{c}{$\begin{array}{c}\text { Result } \\
(\%)\end{array}$} & Interpretation \\
\cline { 1 - 2 } Vagal response & Deep breathing & 74 & \\
\cline { 2 - 3 } & Hand grip & 25 & \\
\cline { 2 - 3 } & Orthostatic test & 20 & \\
\cline { 2 - 3 } Peripheral sympathetic response $\alpha$ & 11 & \multirow{2}{*}{ Preserved peripheral sympathetic response } \\
\cline { 1 - 2 } Peripheral sympathetic response $\beta$ & 12 & \\
\hline Central sympathetic response $\alpha$ & 9 & Central sympathetic deficiency \\
\hline Central sympathetic response $\beta$ & 7 &
\end{tabular}

The normal value is $30 \%$ for the Deep breathing test and $10 \%$ for remaining tests.

The autonomic profile of the patient is in favor of a vagal hyperactivity, as assessed by the three tests that evaluate vagal response (Deep Breathing, Hand grip and Orthostatism), and is responsible of high degree atrioventricular block and ventricular asystole during deep breathing test. Peripheral sympathetic response is preserved and there is a central sympathetic deficiency.

\section{DisCUSSION}

The autonomic nervous system is divided into two components: sympathetic and parasympathetic. The cardiac conduction system is densely innervated by each of these two divisions. The parasympathetic contingent acts by decreasing the contractility of the heart (negative inotropic effect), the heart rate (negative chronotropic effect), and the conduction velocity within the cardiac conduction system (negative dromotropic effect). It innervates the sinus node and the atrioventricular node via the two vagus nerves. The right vagus nerve supplies the sinoatrial (SA) node and slows its pacemaker, while the left vagus innervates the atrioventricular (AV) node [4].

Vagally mediated atrioventricular block is a functional and paroxysmal conduction disorder related to the parasympathetic influence on atrioventricular conduction [5] and is characterized by narrow QRS complexes [6]. It can occur acutely, during stimulation of the parasympathetic system (during vagal 
stimulation, carotid sinus massage, emotion, etc.), or during the recovery phase after physical exercise. Clinically, it may manifest as discomfort or syncope. Vagally mediated atrioventricular block can be chronic and can be observed in young people and in highperformance athletes. In this case, it is asymptomatic and considered physiological.

Cardiovascular exploration of the autonomous nervous system is based on various cardiovascular autonomic tests that are performed while measuring the variability in the heart rate and blood pressure. Among these tests is the deep-breathing test, which is considered the main test for the exploration of parasympathetic function [7, 8]. It explores the variability of heart rate (HR) over 6 cycles of inspiration and deep expiration lasting 5 seconds each. During inspiration, the vagal tone is lifted, and the heart rate rises. The opposite happens on expiration. Measuring this variability allows a quantitative approach to assessing vagal activity. Indeed, the dysautonomic origin of certain conductive disorders related to vagal hyperactivity, such as atrioventricular block, can be revealed thanks to cardiovascular reactivity tests without having to resort to invasive tests.

In our clinical case, the deep-breathing test induced the appearance of high-grade atrioventricular block, which was complicated by asystole, indicating a hyperactivity of the left vagus nerve, which innervates the atrioventricular node. The increased vagal tone slows conduction in the atrioventricular node but do not affect His-Purkinj conduction. Thus, the vagally mediated atrioventricular block is benign because it is localized within the atrioventricular node and not in the His-Purkinj system [6]. It is thus a functional conduction disorder and not an expression of anatomical involvement [6]. On the other hand, our patient remained asymptomatic during the test and follow-up. For these reasons, we believe that there is no indication for pacemaker implantation as stated by the guidelines [9]. We decided to monitor the patient closely and over a long period of time, with the view that if symptoms appear and are clearly attributable to this conduction disorder, pacemaker implantation may be reasonable [10].

In order to treat vagal hyperactivity, two promising molecules were suggested for our patient: phenobarbital and maprotiline. Phenobarbital is a barbiturate, with sedative, hypnotic properties. It has been shown that it has a beneficial effect on the functional disorders of dysautonomia during vagal hyperactivity [11]. It reduces this vagal activity by damping the cortical centers [11, 12]. A preliminary study was conducted at the Autonomic Nervous System Unit of Cardiology Department A [13]. This study involved 16 patients who received phenobarbital at a dose of $30 \mathrm{mg} /$ day for 3 months. We found a reduction in vagal activity in the deep breathing test from
$56.5 \% \pm 31$ to $35.1 \% \pm 10.7$ with a significant $\mathrm{p}=0.02$. We found a similar result for the hand grip test and orthostatism. Maproptilline is an imipraminic antidepressant, non-selective monoamine reuptake inhibitor, Noradrenaline reuptake inhibitor, an anticholinergic and histaminergic, decreases the presynaptic reuptake of noradrenaline and facilitates sympathetic transmission. Its anticholinergic effect contributes to the elevation of the heart rate. In a previous study [14], five patients with major vagal hyperactivity associated with severe symptomatic bradycardia underwent autonomic nervous system exploration after treatment with maprotiline $(50 \mathrm{mg}$ daily for three months). We had observed a spectacular functional improvement, with the regression of all signs (lipothymia malaise, vertigo, headache, etc.). Also, we had noted an increase in the basic heart rate by an average of 10 additional beats and a regression of the vagal hyperactivity by an average decrease of $50 \%$ compared to the initial average value. Thereafter, the invention was published describing a novel application of maprotilline in the treatment of autonomic dysregulation with severe symptomatic bradycardia related to its anticholinergic effect [14].

\section{CONCLUSiON}

This case illustrates the contribution of autonomic nervous system exploration by using cardiovascular reactivity tests, in the detection of dyasautonomic origin (vagal hyperactivity) of a high degree conductive disorder. Future and larger studies are desirable to confirm the encouraging effects of phenobarbital and maprotiline in the treatment of severe vagal hyperactivity.

\section{REFERENCES}

1. Ewing DJ, Martyn CN, Young RJ, Clarke BF. The value of cardiovascular autonomic function tests: 10 years experience in diabetes. Diabetes care. 1985 Sep 1;8(5):491-8.

2. Ewing DJ, Irving JB, Kerr F, Wildsmith JA, Clarke BF. Cardiovascular responses to sustained handgrip in normal subjects and in patients with diabetes mellitus: a test of autonomic function. Clinical science and molecular medicine. 1974 Mar;46(3):295-306.

3. Benarroch PLE. Laboratory evaluation of autonomic function, chez Clinical autonomic disorders (2nd edn), Philadelphia, Pensylvania, USA, Lippincott-Raven Publishers, 179-208.

4. Cheng Z, Powley TL, Schwaber JS, Doyle III FJ. Projections of the dorsal motor nucleus of the vagus to cardiac ganglia of rat atria: an anterograde tracing study. Journal of Comparative Neurology. 1999 Jul 26;410(2):320-41.

5. Aksu T, Guler TE, Bozyel S, Yalin K. Potential usage of cardioneuroablation in vagally mediated functional atrioventricular block. SAGE open medicine. 2019 Mar;7:2050312119836308. 
6. Alboni P, Holz A, Brignole M. Vagally mediated atrioventricular block: pathophysiology and diagnosis. Heart. 2013 Jul 1;99(13):904-8.

7. Baschieri F, Calandra-Buonaura G, Doria A, Mastrolilli F, Palareti A, Barletta G, Solieri L, Guaraldi P, Martinelli P, Cortelli P. Cardiovascular autonomic testing performed with a new integrated instrumental approach is useful in differentiating MSA-P from PD at an early stage. Parkinsonism \& related disorders. 2015 May $1 ; 21(5): 477-82$.

8. Souad A, Hanane R, Leila E, Youssouf R, Latéfa O, Abdellatif A, Azeddine I, Leslie C, Mohammed C, Halima B, Taoufiq D. Young footballers, assessed by deep breathing test, have a higher vagal response. Open Journal of Molecular and Integrative Physiology. 2012 Aug 23;2012.

9. Kusumoto FM. Guideline on the Evaluation and Management of Patients With Bradycardia and Cardiac Conduction Delay: A Report of the American College of Cardiology/American Heart Association Task Force on Clinical Practice Guidelines and the Heart Rhythm Society. Circulation. 2019; 140(18):382-482.
10. Shen WK, Sheldon RS, Benditt DG, Cohen MI, Forman DE, Goldberger ZD, Grubb BP, Hamdan MH, Krahn AD, Link MS, Olshansky B. 2017 ACC/AHA/HRS guideline for the evaluation and management of patients with syncope: a report of the American College of Cardiology/American Heart Association Task Force on Clinical Practice Guidelines and the Heart Rhythm Society. Journal of the American College of Cardiology. $2017 \mathrm{Jul}$ 24;70(5):e39-110.

11. Robertson D. Chez Primer on the Autonomic Nervous System, New York, Academic Press, 2004; 1-386.

12. La monographie du Bellamine, [En ligne]. Available: http://www.drugs.com/ppa/belladonnaphenobarbital-ergotamine-tartrate.html.

13. Hajoui FZ. Effet du phénobarbital à faible dose sur le système nerveux autonome (SNA): Intérêt de l'exploration du système neurovégétatif avant et après traitement (Doctoral dissertation). 2011.

14. Harold CC. Universite Mohammed V Souissi in Rabat. Morocco Brevet WO/2013/154408, 2013. 